\title{
La desnutrición infantil
}

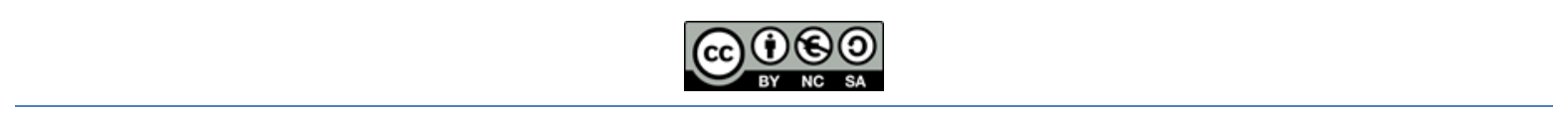

\section{Child malnutrition}

\author{
Yolanda Tatiana Carrasco Ruano. ${ }^{1}$
}

Resumen.

DOI: https://doi.org/10.33262/concienciadigital.v2i2.941

En el ecuador la desnutrición infantil ha sido un factor muy alarmante ante la sociedad y los mismos gobernantes, la desnutrición no es por no haber alimentos sino porque no pueden ser adquiridos por las familias de bajos recursos económicos, la desnutrición y el incremento de la pobreza vienen de la mano lo cual genera un alto grado de reciprocidad en nuestro país, al habla de este problema social no solo nos enfocaremos en nuestro país sino en el resto de las naciones y también en los organismos y organizaciones que han tomado cartas en el asunto para remediar el impacto de la desnutrición si bien es que la economía afecta a casi todos los países del tercer mundo y no pueden generar fuentes de empleo para su población local porque las grandes potencias también sufren de este problema social, cabe recalcar que la desnutrición afecta a todo el mundo y más a los infantes los cuales no pueden valerse por sí mismo es ahí donde este tema es más alarmante por que en los últimos años la desnutrición infantil ha crecido en algunos países y en otros ha descendido, este trabajo tiene un carácter investigativo y comparativo de los países con el alto grado de desnutrición y la evolución que ha tenido nuestro país en este tema.

Palabras claves: Desnutrición, infantes, globalización.

\section{Abstract.}

In Ecuador, child malnutrition has been a very alarming factor before society and the rulers themselves, malnutrition is not because there is no food but because they cannot be acquired by low-income families, malnutrition and increasing poverty They come hand in hand which generates a high degree of reciprocity in our country, speaking of this social problem we will not only focus on our country but on the rest of the nations

\footnotetext{
${ }^{1}$ Ciencia digital, Ambato, Ecuador, tcarrascor@yahoo.com
} 
and also on the organizations and organizations that have taken action on the matter to remedy the impact of malnutrition, although the economy affects almost all third world countries and cannot generate sources of employment for their local population because the great powers also suffer from this social problem, it should be emphasized that malnutrition affects to the whole world and more to the infants who cannot fend for themselves is where this issue is most alarming because in the $\mathrm{u}$ In recent years, child malnutrition has grown in some countries and in others it has declined, this work has an investigative and comparative character of countries with a high degree of malnutrition and the evolution that our country has had in this area.

Keywords: Malnutrition, infants, globalization.

\section{Introducción.}

\section{¿Qué es la desnutrición?}

Andrade, G. (2014), Reyes, M (2018) y UNICEF, (2007) mencionan que la desnutrición es un siclo no biológico que es generado por la falda de alimentos, la desnutrición es mucho más frecuente y con mayor impacto en la población infantil y más en los países del tercer mundo. Desde el nacimiento hasta los 6 meces de edad la lactancia materna le brinda al bebe todos los nutrientes necesarios para su crecimiento normal pero a mayor edad como un año de edad o más la leche materna ya no brinda todos los nutrientes necesarios es ahí donde los alimentos sólidos pueden ser digeridos por el sistema digestivo de todo organismo vivo y si estos alimentos no son ingeridos en la demanda que requiere su apetito y necesidad es donde entra a una pérdida de grasa corporal y masa muscular lo cual se divide por faces, en la primera fase los niños dejan de aumentar de peso, lo cual genera una delgadez notable en el infante, no solo le dan a los niños también le puede coger a personas adultas y a mayores de edad si su alimentación no es buena ni regular, pueden ocasionar problemas gástricos e intestinales. Concluyendo en países de medio oriente y regiones de la áfrico donde la mendicidad y la pobreza abunda 8 de cada 10 infantes en estas regiones sufren de desnutrición crónica y severa las cuales dan un alto grado de mortalidad afectando en la salud por la acumulación de cadáveres en fosas comunes.

La desnutrición es un problema global que ocurre en sectores y países donde los alimentos son escasos, hoy en día la economía y la corrupción han hecho que muchos países de áfrica y sudamericanos sufran una desnutrición infantil crónica y a gran escala lo cual genera problemas de salud organizaciones como la ONU y la UNICEF hay echo campañas y colaboraciones con grandes buques de alimentos no perecibles pero eso no se puede abastecer a toda la población que sufre este mal de la sociedad actual, la desnutrición puede ser hasta mortal llevando al individuo a una pérdida de peso y grasa corporal llevándolo a la muerte. Es por eso que si no se toma cartas en el asunto por parte de los países de Latinoamérica la 
población caería en un círculo cuyos infantes tendrán que afrontar la consecuencia de malos gobernantes.

Figura N 1. Porcentajes de la mala nutrición infantil en el mundo.

\begin{tabular}{|c|c|c|c|c|c|}
\hline \multicolumn{2}{|l|}{ Región de África } & \multicolumn{2}{|c|}{$\begin{array}{l}\text { Región de Asia } \\
\text { Sudoriental }\end{array}$} & \multicolumn{2}{|l|}{ Región de Europa } \\
\hline No. de casos no & No. de casos notificados & \multicolumn{2}{|c|}{ País No. de casos notificados } & \multicolumn{2}{|c|}{ País No. de casos notificados } \\
\hline \multicolumn{2}{|c|}{ República Democrática del Congo 72029} & \multirow{2}{*}{$\begin{array}{l}\text { India } \\
\text { Indonesia }\end{array}$} & \multirow{2}{*}{$\begin{array}{l}18668 \\
15489\end{array}$} & \multicolumn{2}{|c|}{ Ucrania $\quad 12746$} \\
\hline Burkina Faso & 7362 & & & Rumanía & $745 d$ \\
\hline Nigeria & 6447 & Tailandia & 5197 & Federación de Rusia & 2123 \\
\hline Angola & 4458 & Nepal & 3362 & Reino Unido & 2092 \\
\hline Etiopía & 4347 & Myanmar & 2175 & España & 1204 \\
\hline Uganda & 2027 & \multirow[t]{5}{*}{ Bangladesh } & \multirow[t]{5}{*}{1986} & & \\
\hline Sudán del Sur & 1952 & & & & \\
\hline República Unida de Tanzanía & 1668 & & & & \\
\hline Ghana & 1613 & & & & \\
\hline Guinea Ecuatorial & 1190 & & & & \\
\hline \multicolumn{2}{|c|}{ Región del Mediterráneo Oriental } & \multicolumn{2}{|c|}{$\begin{array}{l}\text { Región del Pacífico } \\
\text { Occidental }\end{array}$} & & \\
\hline \multicolumn{2}{|c|}{ No. de casos notificados } & \multicolumn{2}{|c|}{ País No. de casos notificados } & & \\
\hline Somalia & 9983 & China & 6183 & & \\
\hline Sudán & 8523 & Malasia & 1868 & & \\
\hline Pakistán & 8046 & \multirow[t]{3}{*}{ Filipinas } & \multirow[t]{3}{*}{1536} & & \\
\hline Afganistán & 2787 & & & & \\
\hline Yemen & 2177 & & & & \\
\hline
\end{tabular}

Fuente: https://www.eluniverso.com/noticias/2016/09/04/nota/5780076/desnutricioninfantil-mal-que-cuesta-revertir

\section{Resultados.}

En la imagen se muestra que los altos porcentajes de mortalidad infantil se debe a la mala nutrición que reciben y a una pobreza extrema, así es el caso de áfrica y medio oriente cuales son las regiones con asma alta tasa de mortalidad de niños presenta y los números más elevados de desnutrición infantil, quedando en tercer lugar Latinoamérica como foco propenso a que los infantes no sean alimentados bien por la falta de economía en países andinos, recayendo a la mendicidad y otros actos ilegales como es el consumo de estupefacientes.

\section{¿La desnutrición infantil en Latinoamérica?}

ABC Color (Paraguay), (2012) Hervas, V. (2006) y TodosAyudan.es, (2017) mencionan que la desnutrición infantil es un problema mundial y más de 60 millones de niños menores a 4 años la padecen, uno de cada 4 niños sufren de mala nutrición y estos son los que se localizan en zonas rurales y tienen un escaso nivel educacional lo cual ayuda a impedir el alcance a los objetivos del nuevo milenio mencionados por las organizaciones como son la ONU y la UNICEF cuales son los que se han preocupado de este problema social en los lugares que 
más alto porcentaje de desnutrición infantil tienen es así como Latinoamérica entra a este círculo problemático por que en los sectores andinas y costeras hay un alto número de infantes con problemas de desnutrición sebera o crónica lo cual los gobiernos encargados de salvaguardar a la población han pisoteado este derecho humano y pisotean el derecho a una alimentación diaria y constante de los niños, el derecho a una vida justa ese derecho en sectores no es muy tomado encuentra ya que además de sufrir de la desnutrición no tienen alcantarillado, acceso al agua potable y mucho menos a una educación de calidad y calidez.

En Latinoamérica y resto del mundo la desnutrición ha golpeado muy duro en la sociedad actual lo cual ha generado millones de interrogantes las cuales solo pocas organizaciones mundiales han tomado cartas en el asunto, es por eso que el alto número de mala nutrición infantil y el alto número de natalidad generan más infantes con problemas de mala nutrición, cabe mencionar que no solo a los infantes les da desnutrición también a la gente adulta y mucho más a los mayores ancianos los cuales son abandonados y aislados de la sociedad que las rodea como basura dejándolos a su suerte y como ellos ya no pueden solventar sus gastos sufren más y mueren más rápido.

Figura N 2. Desnutrición infantil en Latinoamérica estadísticas.

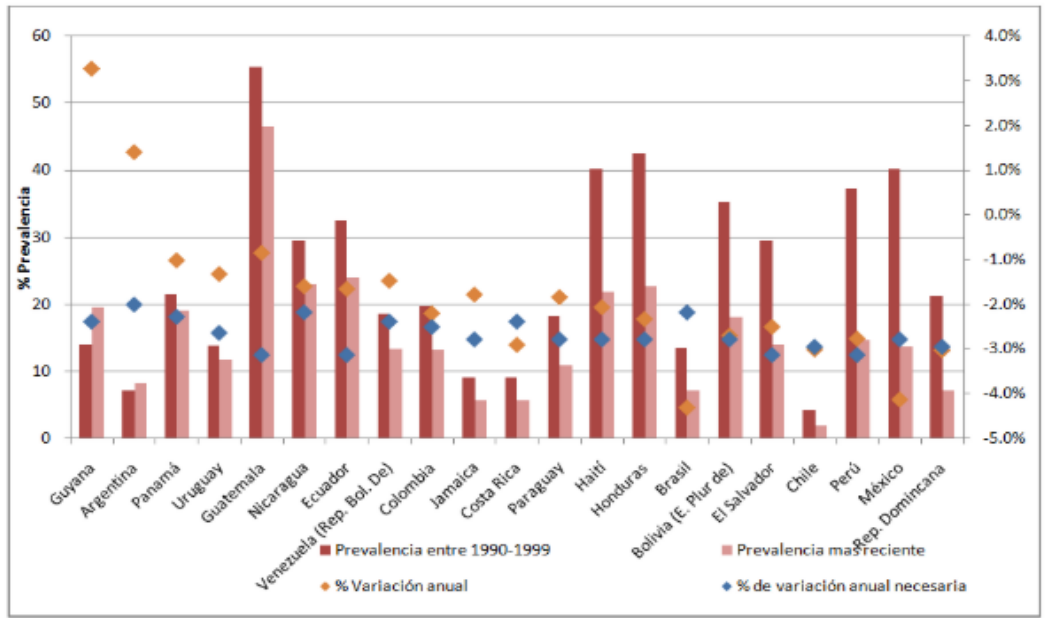

Fuente: http://todosayudan.com/wp-content/uploads/2011/02/ni\%C3\%B1os-5.jpg

\section{Resultados.}

En la imagen se puede denotar a dos infantes uno que aproximadamente tiene una edad de 7 a 8 años y un bebe de meses de nacido con un severo problema de desnutrición, talvez porque la madre o el padre no cuenta con los recursos necesarios para abastecer su hogar de alimentos, la bebe se la nota desorientada con una baja masa muscular donde se le pueden notar hasta sus costillas, se ve que los infantes son de un sector rural y a demás son de un hogar pobre. 


\section{¿La desnutrición infantil en el ecuador?}

Bravo, G. Márquez, R \& Danta, S (2018), América latina, (2016) y Cluster Salud.es, (2016) mencionan que el Ecuador ha pasado por diferentes etapas de este problema social el cual El $26 \%$ de los ecuatorianos menores de cinco años son los que padecen de la desnutrición crónica, es lo que representa una cifra de más de 370.000 niños en todo el país y más de las zonas rurales, según datos del Ministerio de Salud, que alerta sobre algunas provincias como son Esmeraldas, Manabí, Guayas y las regiones amazónicas del territorio con más alto número de infantes con desnutrición. Además, los datos de la secretaría de Salud destacan que la desnutrición crónica afecta en mayor medida a los niños indígenas ya afrodescendientes de las regiones más inaccesibles del territorio, cuyas poblaciones tienen un $63,5 \%$ de desnutridos crónicos entre los menores de cinco años y adultos ancianos. Cabe recalcar que han habido organizaciones no gubernamentales que han ayudado a que el porcentaje de este problema social disminuya considerablemente fomentando así una vida mejor para los infantes.

Luego de más de 30 años contra la batalla de la desnutrición crónica del país las cifras han disminuido gracias a muchas organizaciones humanistas y caritativas que gracias a campañas de salud y alimentación han hecho que tanto niños como adultos mayores puedan tener una vida más digna y sustentable, solo hace falta mantenerla ya que las fuentes de trabajo han disminuido en la actualidad y eso puede perjudicar en la nutrición de los infantes gracias a la llegada de venezolanos los cuales trabajan por menos salario y los ecuatorianos se quedan sin ingresos económicos en el ecuador ya que la mayor parte de las divisas que gana los venezolanos no se quedan aquí sino marchan a su país.

Figura N 3. Muestra del porcentaje de infantes con una desnutrición crónica.

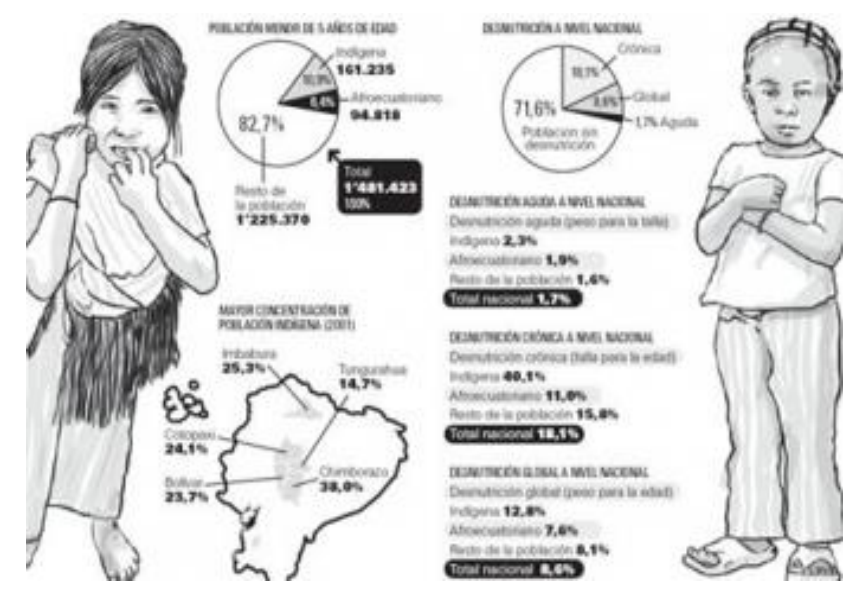

Fuente:http://camilayanez23.blogspot.com/2014/04/la-desnutricion-en-elecuador.html 


\section{Resultados.}

En la imagen se puede notar que la desnutrición infantil cae más sobre los niños de poblaciones indígenas y afrodescendientes ya que la economía de ellas y el sector donde se localizan hace difícil una buena nutrición los porcentajes son muy elevados y alarmantes para la sociedad. Es así que la población en el ecuador ha ido aumentando por que aún existen familias disconforme y sin un sustento económico regular para poder solventar las necesidades básicas de cada integrante del círculo familiar.

\section{¿Qué problemas genera la desnutrición infantil?}

caritasarquidiocesana.org, (2015) y wikis, (2014) mencionan que la desnutrición infantil es una enfermedad que afecta sobre todo a los niños, niñas menores de cinco años y mayores de edad, este problema es provocada por la falta de alimentos y las recurrentes enfermedades que no les permite un buen ingreso biológico de los alimentos que ingieren. Entre las causas de desnutrición podemos mencionar la falta de alimentos en casa ya sea por baja disponibilidad o acceso, catástrofes naturales que obligan a las familias a abandonar sus hogares, suspensión de lactancia materna exclusiva a niños y niñas menores de 5 a 7 meses de edad por falta de ingesta alimentaria y nutricional de la madre, enfermedades como el SIDA, tuberculosis, sarampión, parasitosis graves, problemas políticos como guerras. Entre la mayoría de factores que facilitan la desnutrición factores de riesgo podemos hallar problemas de ingresos en las familias desempleo, problemas de cosechas, etcétera, falta de higiene agua sin tratar, falta de letrinas, alimentos mal preparados, y demás, enfermedades comunes diarreas, fiebres, neumonías, bajo nivel social y económico, alimentación inadecuada o monótona y problemas sociales.

Figura N 4. Porcentajes de mendicidad en el ecuador.

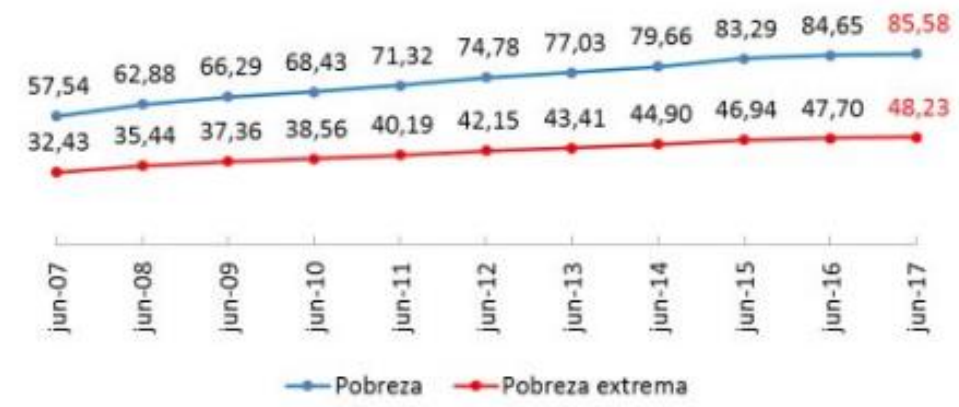

Fuente: ENEMDU

Nota (1): Los datos se encuentran expresados en dolares mensuales corrientes.

Fuente: https://caritasarquidiocesana.org/desnutricion-causas-consecuencias/ 


\section{Resultados.}

Podemos ver que la mendicidad y la pobreza que algunas familias es la que genera en los infantes la desnutrición sea crónica o severa.

Las consecuencias de la desnutrición, durante la infancia y edad preescolar se asocian con retardo en el crecimiento y desarrollo psicomotor, además se puede encontrar un aumento de las enfermedades por descenso del sistema inmunológico, déficit de vitaminas necesarias para el buen funcionamiento del organismo y minerales, deshidratación, hipoglicemia, hipotermia o incluso hasta la muerte. A largo plazo la desnutrición puede llevarnos a disminución en la capacidad de trabajo físico y desempeño escolar en la edad escolar, la adolescencia y la edad adulta por lo que se aumenta la pobreza. Incluso aumenta la propensión a enfermedades como obesidad, diabetes, hipertensión, también dislipidemias entro otros efectos metabólicos a largo plazo. Más aún, la desnutrición durante los primeros años de vida, a través de sus efectos adversos en el crecimiento durante la infancia de las mujeres, tiene efectos negativos en el peso al nacer la siguiente generación.

La desnutrición infantil genera millones de problemas tanto de salud como social los cuales alarman a la sociedad y la mayoría de los infantes que la padecen no sobreviven y mueren, el SIDA, la tuberculosis y los problemas gástricos generan que la desnutrición se agrave e incremente más dando una sobre explotación de centros de salud.

\section{Conclusiones}

- En concreción la desnutrición es una falta de alimento en el organismo generando enfermedades de factor grave y crónico, en si la desnutrición se ve afectada más en la población infante y adulto mayor los cuales no pueden depender ni solventarse, la desnutrición se ve acompañada de la mala economía de un hogar y de la falta de ayuda por parte de los mandatarios de un país.

- En el ecuador la desnutrición en los años 90 era muy grave y su alto grado de desnutrición que padecían y más los sectores rurales han hecho que la tasa de mortalidad haya incrementado, la falta de una economía sustentable en el país y al no generarse fuentes de trabajo han ocasionado la mala nutrición infantil, actualmente este problema ha descendido en cifras muy considerables que han hecho al ecuador un precursor para seguir como ejemplo páralas demás naciones en el combate de la desnutrición crónica infantil.

- Las enfermedades más comunes que conlleva la desnutrición son tuberculosis, problemas intestinales, parásitos, gastritis y el sida hay organizaciones que han hecho que la desnutrición infantil este más factible remediar en una nación. El generar fuentes de empleo podemos ayudar en la buena nutrición de los infantes y reducir la mendicidad y otros problemas sociales que vienen sujeta de la mano por parte de la pobreza. 
- Hoy en día las organizaciones como son la ONU y la UNICEF han tomado cartas en el asunto y desean generar planes de contingencia para que este problema social no aumente, generando fuentes de empleo, dotando de alimentos a sectores y países que han sufrido alguna catástrofe entre otras opciones que podrían ayudar a combatir la desnutrición infantil

\section{Referencias bibliográficas.}

ABC Color (Paraguay). (10 de 06 de 2012). ABC Color (Paraguay). Recuperado el 29 de 10 de 2018, de Desnutrición en Latinoamérica: https://www.americaeconomia.com/politica-sociedad/sociedad/la-desnutricioninfantil-un-grave-problema-que-afectaria-el-desarrollo-de

América latina. (28 de 07 de 2016). amerlat.ec. Recuperado el 29 de 10 de 2018, de desnutricion infantil en el ecuador: https://mundo.sputniknews.com/americalatina/201607281062517589-ecuadordesnutricion-infantil/

Andrade, M. P. (22 de 07 de 2014). Definicion ABC. Recuperado el 29 de 10 de 2018, de desnutricion: https://www.definicionabc.com/?s=desnutricion

Bravo, D., Márquez, C., \& Data, E. C. (21 de 01 de 2018). El universo. Recuperado el 29 de 10 de 2018, de desnutricion en ecuador: https://www.elcomercio.com/actualidad/desnutricion-infantil-erradicacion-ecuadorproblemas.html

Cluster Salud.es. (05 de 09 de 2016). Cluster Salud. Recuperado el 29 de 10 de 2018, de ecuador y salud : https://clustersalud.americaeconomia.com/ecuador-35-las-muertesmenores-5-anos-ocurre-desnutricion

Hervas, M. (03 de 05 de 2006). Còrdova.en. Recuperado el 29 de 10 de 2018, de desnutricion infantil: https://www.diariocordoba.com/noticias/sociedad/desnutricion-afectamundo-uno-cuatro-menores-5-anos_247214.html

Reyes, M. (14 de 06 de 2018). Nutricion.ec. Recuperado el 29 de 10 de 2018, de desnutricion: http://www.nutricion.ec/index.php/blog.html

TodosAyudan.es. (04 de 10 de 2017). TodosAyudan.es. Recuperado el 28 de 10 de 2018, de Desnutricion infantil en latinoamerica: http://www.todosayudan.com/ladesnutricion-infantil-una-de-las-principales-causa-de-pobreza/

UNICEF. (22 de 05 de 2007). UNICEF.com. Recuperado el 29 de 10 de 2018, de Desnutricion:

https://www.unicef.org/republicadominicana/health_childhood_10172.htm

https://caritasarquidiocesana.org/desnutricion-causas-consecuencias/ 
http://camilayanez23.blogspot.com/2014/04/la-desnutricion-en-el-ecuador.html

https://www.eluniverso.com/noticias/2016/09/04/nota/5780076/desnutricion-infantil-malque-cuesta-revertir

http://todosayudan.com/wp-content/uploads/2011/02/ni\%C3\%B1os-5.jpg

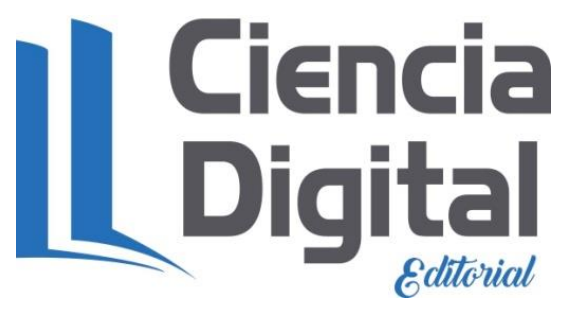


Para citar el artículo indexado.

Carrasco Ruano, Y. (2019). La desnutrición infantil. ConcienciaDigital, 2(2), 17-26. https://doi.org/10.33262/concienciadigital.v2i2.941

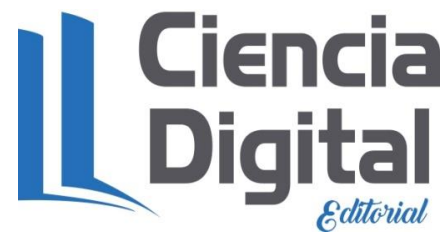

El artículo que se publica es de exclusiva responsabilidad de los autores y no necesariamente reflejan el pensamiento de la Revista Conciencia Digital.

El articulo queda en propiedad de la revista y, por tanto, su publicación parcial y/o total en otro medio tiene que ser autorizado por el director de la Revista Conciencia Digital.

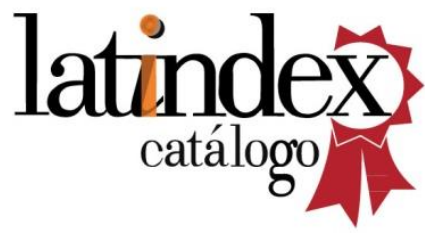

Conciencia

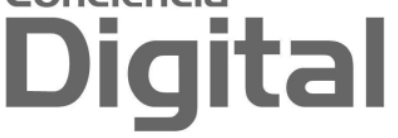

